University of Wollongong

Research Online

Faculty of Engineering and Information

Faculty of Engineering and Information

Sciences - Papers: Part A

Sciences

$1-1-2016$

Pricing European options with stochastic volatility under the minimal entropy martingale measure

Xin-Jiang He

University of Wollongong, xh016@uowmail.edu.au

Song-Ping Zhu

University of Wollongong, spz@uow.edu.au

Follow this and additional works at: https://ro.uow.edu.au/eispapers

Part of the Engineering Commons, and the Science and Technology Studies Commons

Research Online is the open access institutional repository for the University of Wollongong. For further information contact the UOW Library: research-pubs@uow.edu.au 


\title{
Pricing European options with stochastic volatility under the minimal entropy martingale measure
}

\author{
Abstract \\ In this paper, a closed-form pricing formula in the form of an infinite series for European call options is \\ derived for the Heston stochastic volatility model under a chosen martingale measure. Given that markets \\ with the stochastic volatility are incomplete, there exists a number of equivalent martingale measures and \\ consequently investors face a problem of making a choice of appropriate measure when they price \\ options. The one we adopt here is the so-called minimal entropy martingale measure shown to be related \\ to the expected utility maximization theory (Frittelli 2000 Math. Finance 10(1), 39-52) and the financial \\ rationality for choosing this measure will be further illustrated in this paper. A great advantage of our \\ newly-derived pricing formula is that the convergence of the solution in series form can be proved \\ theoretically; such a proof of the convergence is also complemented by some numerical examples to \\ demonstrate the speed of convergence. To further show the validity of our formula, a comparison of \\ prices calculated through the newly derived formula is made with those obtained directly from the Monte \\ Carlo simulation as well as those from solving the PDE (partial differential equation) with the finite \\ difference method.

\section{Disciplines} \\ Engineering | Science and Technology Studies

\section{Publication Details} \\ He, X. \& Zhu, S. (2016). Pricing European options with stochastic volatility under the minimal entropy \\ martingale measure. European Journal of Applied Mathematics, 27 (2), 233-247.
}




\title{
Pricing European options with stochastic volatility under the minimal entropy martingale measure
}

\author{
Xin-Jiang $\mathrm{HE}^{*} \quad$ Song-Ping ZHU ${ }^{\dagger}$
}

\begin{abstract}
In this paper a closed-form pricing formula in the form of an infinite series for European call options is derived for the Heston stochastic volatility model under a chosen martingale measure. Given that markets with the stochastic volatility are incomplete, there exists a number of equivalent martingale measures and consequently investors face a problem of making a choice of appropriate measure when they price options. The one we adopt here is the so-called minimal entropy martingale measure shown to be related to the expected utility maximization theory [12] and the financial rationality for choosing this measure will be further illustrated in this paper. A great advantage of our newly-derived pricing formula is that the convergence of the solution in series form can be proved theoretically; such a proof of the convergence is also complemented by some numerical examples to demonstrate the speed of convergence. To further show the validity of our formula, a comparison of prices calculated through the newly derived formula is made with those obtained directly from the Monte Carlo simulation as well as those from solving the PDE (partial differential equation) with the finite difference method.
\end{abstract}

\section{AMS(MOS) subject classification.}

* School of Mathematics and Applied Statistics, University of Wollongong, NSW 2522, Australia.

†Corresponding author: School of Mathematics and Applied Statistics, University of Wollongong, NSW 2522, Australia 
Keywords. Series expansion, Minimal entropy martingale measure, Expected utility maximization, Convergence.

\section{Introduction}

Although Black \& Scholes [5] made a great contribution to the area of option pricing by proposing a simple and closed-form pricing formula for European options in 1973, their assumption for the log-returns of the underlying asset to follow a normal distribution proves to be an over simplification in some cases (it can not capture the features like skewness [21] and fat-tails [23] exhibited by the real market data), which may lead to the problem of mispricing. As a result, many modifications to the Black-Scholes model have been proposed, including one of the most well-known ways by relaxing the assumption of the constant volatility due to the observed "volatility smile" from market data [10].

In the literature, there are mainly two kinds of non-constant volatility models, i.e. local volatility and stochastic volatility model. Local volatility models were adopted by Dupire [11], Derman \& Kani [9] and Rubinstein [24] and the volatility in their models was defined as a deterministic function of asset price and time. Although this kind of model appears to have certain advantages since it may relieve some computational burden and fit well with a given market, it was shown that local volatility model is not as flexible as the stochastic volatility models [10] and it is even suggested in Hagan et. al [14] that the "smile dynamics" are poorly predicted by the local volatility model. Such kind of observations have made stochastic volatility models much more popular over local volatility models.

Stochastic volatility was first systematically studied by Scott [28] who numerically solved the option pricing problem with Monte Carlo simulations. It was further investigated by Hull \& White [17] and Stein \& Stein [31]; the former proposed a series approximation solution and the latter presented a closed-form formula for European option price. Unfortunately, their models are not satisfying since some drawbacks do exist. For example, 
the stochastic volatility process of Hull \& White model does not possess the mean-reverting property, which is at odds with observations from many empirical studies [2], while the volatility can not always remain positive under some parameter settings of Stein \& Stein model.

A breakthrough took place in 1993 when Heston [15] incorporated a specific model with the stochastic volatility possessing the mean-reverting and non-negative properties and derived a closed-form pricing formula based on the Fourier transform technique. However, despite its great success, one should notice that the pricing formula obtained by Heston is not the only price formula under the proposed model. This is because the market described by the Heston model is actually incomplete as a result of introducing stochastic volatility and thus derivative pricing is no longer independent of investors' risk preference. Thus, different choice of equivalent martingale measures will lead to different risk-neutral prices. There are then two issues one may face; a) we need a reasonable principle to select an appropriate martingale measure, and b) we need to overcome the difficulty of deriving a closed-form pricing formula associated with the chosen martingale measure.

In fact, there are several different martingale measures, such as minimal martingale measure [25], variance-optimal measure [20, 26], minimal reverse entropy martingale measure [27] and so on. The minimal entropy martingale measure considered here is one of them and is chosen by minimizing the relative entropy between the pricing measures and the physical measure. A financial interpretation for choosing the minimal entropy martingale measure was originally given in [12], which is related to the concept of expected utility maximization, and will be further illustrated later in this paper showing that choosing the minimal entropy martingale measure is equivalent to attaining the maximum expected utility, an action axiom in finance. Unfortunately, to the best of our knowledge, only numerical methods are applied to option pricing under this particular measure, and a closed-form pricing formula has hitherto not been found. A great advantage of having analytical closed-form formula in terms of option pricing is that considerable amount of 
time and effort can be spared when these formula are involved in the determination of model parameters; a feature that none of numerical pricing approaches could match. This is the main thrust behind our effort of searching for a closed-form solution of the Heston stochastic volatility model under the minimal entropy martingale measure.

Entropy is a concept originated from thermodynamics [7] and it has been applied to portfolio selection and asset pricing by a number of different authors, such as Philippatos \& Wilson [22], who were believed to be the first to apply this concept to portfolio selection and adopted it as a measure of risk, and Buchen \& Kelly [6], who estimated the distribution of the underlying from a set of option prices with the principle of maximum entropy. In contrast, we adopt the concept but use it in the context of pricing options in an incomplete market. Let us consider a stochastic process $S=\left(S_{t}\right)_{t \geq 0}$ defined on a probability space $(\Omega, \mathcal{F}, \mathrm{P})$ and adapted to the filtration $\boldsymbol{F}=\left(\mathcal{F}_{t}\right)_{t \geq 0}$. The relative entropy $H(Q, P)$ of an equivalent martingale measure $Q$ for $S$ is then defined as

$$
H(Q, P)= \begin{cases}E\left[\frac{d Q}{d P} \ln \left(\frac{d Q}{d P}\right)\right], & Q \ll P \\ +\infty, & \text { otherwise }\end{cases}
$$

where $E$ represents the expectation operator under measure $P$. We denote $M$ as the set of martingale measures and $Q_{e}$ as the minimal entropy martingale measure satisfying

$$
H\left(Q_{e}, P\right)=\min _{Q \in M} H(Q, P) .
$$

It is proved by Frittelli [12] that a unique minimal entropy martingale measure equivalent to $P$ exists under the assumption that there is an equivalent martingale measure $Q_{0}$ such that $H\left(Q_{0}, P\right)<+\infty$. Under this assumption, Hobson [16] managed to obtain an explicit expression of the dynamics for the underlying price and the volatility under the minimal entropy martingale measure with some particular settings. Following him, we take zero interest rate as a leading order approximation. This is a reasonable approximation because 
the tenor of most exchange-traded options is often less than three months, which means the risk-free interest rate (often approximated by Treasury Bill Rates) should be quite low [1], and the changes in the value of the interest rate has little influence on option prices [30]. In this case, although the new PDE (partial differential equation) system governing the option price contains variable coefficients as a result of the introduction of time-dependent drift term in the volatility process, which has made the task of finding closed-form solutions much more difficult, we still managed to find a closed-form pricing formula in series form together with a lower bound for the radius of convergence. In addition, two numerical examples are provided to further demonstrate the speed of convergence of the series solution. To further validate the newly derived pricing formula, we have also carried out additional comparison of the results produced with the new formula and those directly obtained from solving the PDE with the finite difference method and from solving the SDE (stochastic differential equation) with Monte Carlo simulation.

The rest of the paper is organized as follows. In Section 2, we will provide the financial meaning of the minimal entropy martingale measure. In Section 3, we will first briefly introduce the model we adopt and then derive a series solution to our PDE governing the European option price. Also, the convergence of our solution will be proved theoretically by giving a lower bound for the radius of convergence. In Section 4, the speed of convergence will be tested numerically, after which our formula is further verified by making comparison with the results obtained by the finite difference method and Monte Carlo simulation. Concluding remarks are given in the last section.

\section{Financial explanation for minimal entropy martin- gale measure}

In this section, we will briefly review the relationship between minimizing the relative entropy and maximizing the expected exponential utility, which is actually one of the most 
important areas of research on the minimal entropy martingale measure.

According to the existing literature, Frittelli [12] and Bellini \& Frittelli [3] provided an elaborate illustration about the link between the minimal entropy martingale measure and maximum expected utility. By setting $w_{T}$ be the attainable terminal wealth and the utility function be exponential, i.e.

$$
u(x)=-e^{-x}
$$

the results were presented as

$$
H(Q, P)=\sup _{w_{T} \in L^{\infty}: e^{-r T} E_{Q}\left(w_{T}\right) \leq 0} u^{-1}\left(E_{P}\left[u\left(w_{T}\right)\right]\right),
$$

which implies that the relative entropy is indeed the maximum certainty equivalent [19], $u^{-1}\left(E_{P}\left[u\left(w_{T}\right)\right]\right)$, of attainable wealth $w_{T}$ with prices less than or equal to zero. Then the characteristics of the density of the minimal entropy martingale measure were given, showing the equivalence between maximization of the expected utility and adopting the minimal entropy martingale measure. In addition, Delbaen et. al [8] obtained a more generalized result, which considers a self-financing strategy and an extra contingent claim, further demonstrating that maximizing the expected utility is equivalent to minimizing the relative entropy with an exponential utility function specified as

$$
u(x)=1-e^{-\alpha x}, \quad \alpha>0 .
$$

Moreover, how to construct an optimal strategy for a utility optimization problem through the dual problem of martingale measures was also documented in [33]. It should also be noticed that the minimal entropy martingale measure is quite useful when pricing contingent claims with utility indifference approaches [18, 32].

After the financial justification for choosing the minimal entropy martingale measure is illustrated, we are now ready to price European call options under this particular martingale 
measure, which will be presented in the next Section.

\section{Closed-form formula}

In this section we will firstly specify our model under the physical measure and then pricing dynamics will be proposed by means of measure transformation. After the model is established, the derivation of a closed-form pricing formula for European call options is provided based on the series expansion.

Let $S_{t}$ be the underlying asset price and $v_{t}$ be the volatility, the dynamics under the physical measure are specified as follows

$$
\begin{aligned}
\frac{d S_{t}}{S_{t}} & =\mu v_{t} d t+\sqrt{v_{t}} d B_{t} \\
d v_{t} & =k\left(\theta-v_{t}\right) d t+\beta \sqrt{v_{t}} d W_{t}
\end{aligned}
$$

where $B_{t}$ and $W_{t}$ are two standard Brownian motions with correlation $\rho$. It is obvious that this market is incomplete, where a claim can not be perfectly replicated, and there exists a set of equivalent martingale measures and correspondingly arbitrage-free prices. As a result, we need to choose an appropriate equivalent martingale measure for pricing contingent claims. Over the years, many principles have been developed, such as the minimal martingale measure [25], under which the price of "risk premium" is zero, and varianceoptimal measure $[20,26]$, which is connected with quadratic utility function. The minimal entropy martingale measure adopted in this paper is reasonable to choose according to the utility maximization argument in Section 2. Furthermore, no one has derived a closed-form solution under this measure. As a result, following [16], if we set

$$
W_{t}=\rho B_{t}+\sqrt{1-\rho^{2}} C_{t}
$$

where $B_{t}$ and $C_{t}$ are two independent Brownian motions, the dynamics under the minimal 
entropy martingale measure $Q$ can be obtained by the following transformation

$$
\begin{aligned}
d B_{t}^{Q} & =d B_{t}+\mu v \sqrt{v_{t}} d t \\
d C_{t}^{Q} & =d C_{t}+\frac{1}{\beta \sqrt{1-\rho^{2}}} \lambda(\tau) \sqrt{v_{t}} d t
\end{aligned}
$$

where $\tau=T-t$ and $\lambda(\tau)=2 \Delta \tanh (\Delta \tau+b)-k-\rho \beta \mu$. Here $\Delta=\sqrt{\frac{1}{4} k^{2}+\frac{1}{2} k \rho \beta \mu+\frac{1}{4} \beta^{2} \mu^{2}}$ and $b=\tanh ^{-1}\left(\frac{1}{2} \frac{k+\beta \rho \mu}{\Delta}\right)$. Therefore, the corresponding expression for the dynamics can be derived as

$$
\begin{aligned}
\frac{d S_{t}}{S_{t}} & =\sqrt{v_{t}} d B_{t}^{Q}, \\
d v_{t} & =\left[k\left(\theta-v_{t}\right)-\beta \rho \mu v_{t}-\lambda(\tau) v_{t}\right] d t+\beta \sqrt{v_{t}} d W_{t}^{Q} .
\end{aligned}
$$

Let $U(S, v, t)$ be the European call option price written on the underlying asset $S_{t}$, then according to the Feynman-Kac theorem [29], we can easily show that $U(S, v, t)$ satisfies the PDE

$$
\begin{aligned}
\frac{1}{2} v S^{2} \frac{\partial^{2} U}{\partial S^{2}} & +\rho \sigma v S \frac{\partial^{2} U}{\partial S \partial v}+\frac{1}{2} \sigma^{2} v \frac{\partial^{2} U}{\partial v^{2}} \\
& +[k(\theta-v)-\beta \rho \mu v-\lambda(\tau) v] \frac{\partial U}{\partial v}+\frac{\partial U}{\partial t}=0
\end{aligned}
$$

with terminal condition:

$$
U(S, v, T)=\max (S-K, 0)
$$

and boundary conditions:

$$
\begin{aligned}
& U(0, v, t)=0, \quad \lim _{S \rightarrow+\infty} U(S, v, t)=S, \\
& U(S, \infty, t)=S, \quad \lim _{v \rightarrow 0} U(S, v, t)=\max (S-K, 0) .
\end{aligned}
$$

Here, the reason for the option price to be equal to the payoff function at $v=0$ is illustrated 
in [34]. On the other hand, $U(S, v, t)$ can also be equivalently calculated as

$$
\begin{aligned}
U(S, v, t) & =e^{-r(T-t)} E^{Q}\left[\left(S_{T}-K\right)^{+} \mid S_{t}=S\right], \\
& =S E^{Q}\left[e^{-r(T-t)} \frac{S_{T}}{S} I_{\left\{S_{T}>K\right\}} \mid S_{t}=S\right]-K e^{-r(T-t)} E^{Q}\left[I_{\left\{S_{T}>K\right\}} \mid S_{t}=S\right] .
\end{aligned}
$$

To seek a solution in an affine form later, we can let $U(S, v, t)$ take the form of

$$
U(S, v, t)=S P_{1}(x, v, t)-K e^{-r(T-t)} P_{2}(x, v, t) .
$$

Substituting Equation (3.5) into (3.3) and applying transformation of $x=\ln (S)$ yield the following PDE

$$
\begin{aligned}
\frac{1}{2} v \frac{\partial^{2} P_{j}}{\partial x^{2}} & +\rho \sigma v \frac{\partial^{2} P_{j}}{\partial x \partial v}+\frac{1}{2} \sigma^{2} v \frac{\partial^{2} P_{j}}{\partial v^{2}}+l_{j} v \frac{\partial P_{j}}{\partial x} \\
& +\left[k(\theta-v)-\beta \rho \mu v-\lambda(\tau) v+m_{j} v\right] \frac{\partial P_{j}}{\partial v}+\frac{\partial P_{j}}{\partial t}=0
\end{aligned}
$$

for $j=1,2$, where $l_{1}=\frac{1}{2}, l_{2}=-\frac{1}{2}, m_{1}=\rho \sigma, m_{2}=0$. Now the terminal conditions for $P_{j}$ become

$$
P_{j}(x, v, T ; \ln [K])=I_{\{x \geq \ln [K]\}} .
$$

Let us denote $y=\ln [K]$ and assume that $f_{j}(x, v, t ; \phi)$ be the conditional characteristic function of $x_{T}$, i.e.

$$
f_{j}(x, v, t ; \phi)=E^{Q}\left[e^{i \phi x_{T}} \mid x_{t}=x, v_{t}=v\right] .
$$

It is not difficult to deduce that $f_{j}(x, v, t ; \phi)$ satisfies the same PDE as $P_{j}(x, v, t ; y)$ and thus we obtain

$$
\begin{aligned}
\frac{1}{2} v \frac{\partial^{2} f_{j}}{\partial x^{2}} & +\rho \sigma v \frac{\partial^{2} f_{j}}{\partial x \partial v}+\frac{1}{2} \sigma^{2} v \frac{\partial^{2} f_{j}}{\partial v^{2}}+l_{j} v \frac{\partial f_{j}}{\partial x} \\
& +\left[k(\theta-v)-\beta \rho \mu v-\lambda(\tau) v+m_{j} v\right] \frac{\partial f_{j}}{\partial v}+\frac{\partial f_{j}}{\partial t}=0
\end{aligned}
$$


with known terminal condition $f_{j}(x, v, T ; \phi)=e^{i \phi x}$. Considering the results in [15], it is natural for us to assume that there exists an affine structure solution to PDE (3.8) as

$$
f_{j}=e^{C(\tau ; \phi)+D(\tau ; \phi) v+i \phi x} .
$$

Hereafter, $i$ denotes the imaginary unit. Therefore, by substituting (3.9) into (3.8), we can obtain the following equation after some calculation

$$
\left\{\frac{1}{2} \sigma^{2} D^{2}+\left[i \rho \sigma \phi-k+m_{j}-\beta \rho \mu-\lambda(\tau)\right] D+\left(l_{j} i \phi-\frac{1}{2} \phi^{2}\right)-D^{\prime}(\tau)\right\} v+\left[k \theta D-C^{\prime}(\tau)\right]=0,
$$

which implies that the coefficients of $v^{n}$ should be set to be zero for every $n$ respectively since $v$ is arbitrary. As a result, we have the following two ODEs (ordinary differential equations)

$$
\begin{aligned}
D^{\prime}(\tau) & =\frac{1}{2} \sigma^{2} D^{2}+\left[i \rho \sigma \phi-k+m_{j}-\beta \rho \mu-\lambda(\tau)\right] D+\left(l_{j} i \phi-\frac{1}{2} \phi^{2}\right), \\
C^{\prime}(\tau) & =k \theta D,
\end{aligned}
$$

with initial condition $C(0)=D(0)=0$. It is obvious that if we can derive the solution of $D(\tau ; \phi)$, then $C(\tau ; \phi)$ will be obtained straightforwardly by direct integration. As a result, what we need to do is to focus on $D(\tau ; \phi)$. Actually, it should be noticed that ODE (3.11) is actually a Riccati Equation with variable coefficients, which brings extra burden in finding a closed-form solution for it.

We set $q_{0}=l_{j} i \phi-\frac{1}{2} \phi^{2}, q_{1}=i \rho \sigma \phi-k+m_{j}-\beta \rho \mu-\lambda(\tau)$ and $q_{2}=\frac{1}{2} \sigma^{2}$. After this crucial change of the variables, it is easy to find that $q_{1}$ can be further simplified as

$$
q_{1}=i \rho \sigma \phi+m_{j}-2 \Delta \tanh (\Delta \tau+b)
$$

By applying the transformation of $D=-\frac{u^{\prime}}{q_{2} u}$, the Riccati equation becomes a second-order 
linear ODE with variable coefficients as

$$
u^{\prime \prime}-q_{1} u^{\prime}+q_{0} q_{2} u=0
$$

with $u^{\prime}(0)=0$. Although this kind of ODE can be easily solved when its coefficients are all constant, it still poses an obstacle when its coefficients are a function of the variable $\tau$ as the case we end up with in (3.13). By setting

$$
u=\sum_{n=0}^{\infty} a_{n} \tau^{n}
$$

we obtain

$$
\begin{aligned}
u^{\prime} & =\sum_{n=0}^{\infty}(n+1) a_{n+1} \tau^{n}, \\
u^{\prime \prime} & =\sum_{n=0}^{\infty}(n+1)(n+2) a_{n+2} \tau^{n} .
\end{aligned}
$$

What we need to do now is to expand $\tanh (\Delta \tau+b)$ as a series with respect to $\tau$. However, the coefficients of the series would be very complicated and the property of convergence can hardly be studied as far as the Taylor series for $\tanh (x)$ is concerned, which is a major reason for us to seek alternative ways.

Since $e^{x}$ can be expanded as

$$
e^{x}=\sum_{n=0}^{\infty} \frac{x^{n}}{n !},
$$

and the RHS (right hand side) of Equation (3.16) is a power series, whose radius of convergence for any given $x$ can be calculated as

$$
\lim _{n \rightarrow \infty}\left|\frac{\frac{1}{n !} x^{n}}{\frac{1}{(n+1) !} x^{n+1}}\right|=\lim _{n \rightarrow \infty}\left|\frac{n+1}{x}\right|=+\infty,
$$


we rewrite $\tanh (x)$ as

$$
\tanh (x)=\frac{e^{2 x}-1}{e^{2 x}+1}
$$

since Equation (3.17) means that Equation (3.16) holds for any $x$. As a result, ODE (3.13) can be rearranged as

$$
\left(e^{2 b} \cdot e^{2 \Delta \tau}+1\right) u^{\prime \prime}-\left[\left(i \rho \sigma \phi+m_{j}-2 \Delta\right) e^{2 b} e^{2 \Delta \tau}+\left(i \rho \sigma \phi+m_{j}+2 \Delta\right)\right] u^{\prime}+\left(e^{2 b} \cdot e^{2 \Delta \tau}+1\right) q_{0} q_{2} u=0 .
$$

According to Equation (3.16) we know that

$$
e^{2 \Delta \tau}=\sum_{n=0}^{\infty} c_{n} \tau^{n}
$$

where $c_{n}=\frac{1}{n !}(2 \Delta)^{n}$. Therefore, applying Equation (3.15) and (3.20) into (3.19) yields

$$
\begin{aligned}
& \left(e^{2 b} \sum_{n=0}^{\infty} c_{n} \tau^{n}+1\right) \sum_{n=0}^{\infty}(n+1)(n+2) a_{n+2} \tau^{n}+\left(e^{2 b} \sum_{n=0}^{\infty} c_{n} \tau^{n}+1\right) q_{0} q_{2} \sum_{n=0}^{\infty} a_{n} \tau^{n} \\
& -\left[\left(i \rho \sigma \phi+m_{j}-2 \Delta\right) e^{2 b} \sum_{n=0}^{\infty} c_{n} \tau^{n}+\left(i \rho \sigma \phi+m_{j}+2 \Delta\right)\right] \sum_{n=0}^{\infty}(n+1) a_{n+1} \tau^{n}=0
\end{aligned}
$$

As Equation (3.21) should hold for any $\tau$, the coefficients of $\left\{\tau^{k}, \forall k \geq 0\right\}$ should be equal to zero and thus we have

$$
(k+1)(k+2) a_{k+2}+e^{2 b} \sum_{i=0}^{k}\left[(k-i+2)(k-i+1) a_{k-i+2} c_{i}\right]+I_{2}-I_{1}=0,
$$

for $k \geq 0$, where

$$
\begin{aligned}
& I_{1}=\left\{\left(i \rho \sigma \phi+m_{j}-2 \Delta\right) e^{2 b} \sum_{i=0}^{k}\left[(k-i+1) a_{k-i+1} c_{i}\right]+\left(i \rho \sigma \phi+m_{j}+2 \Delta\right)(k+1) a_{k+1}\right\}=0, \\
& I_{2}=e^{2 b} q_{0} q_{2} \sum_{i=0}^{k}\left(a_{k-i} c_{i}\right)+q_{0} q_{2} a_{k} .
\end{aligned}
$$


As a result, $\left\{a_{k+2}, k \geq 0\right\}$ can be easily obtained once $\left\{a_{n}, n=0,1,2, . ., k+1\right\}$ according to the following equation derived from Equation (3.22)

$$
a_{k+2}=\frac{I_{1}-I_{2}-e^{2 b} \sum_{i=1}^{k}\left[(k-i+1)(k-i+2) a_{k-i+2} c_{i}\right]}{\left(e^{2 b}+1\right)(k+2)(k+1)}, \quad k \geq 0 .
$$

One may think that $D(\tau ; \phi)$ has been solved by now. However, we only have $a_{1}=0$ from $u^{\prime}(0)=D(0)=0$, which keeps $\left\{a_{k+2}, k \geq 0\right\}$ still unknown without the value of $a_{0}$. Fortunately, $D(\tau ; \phi)$ takes the form as

$$
D(\tau)=-\frac{1}{q_{2}} \cdot \frac{\sum_{n=0}^{\infty}(n+1) a_{n+1} \tau^{n}}{\sum_{n=0}^{\infty} a_{n} \tau^{n}}
$$

which means that we do not have to derive $a_{n}$ since $D(\tau)$ can be further represented by

$$
D(\tau)=-\frac{1}{q_{2}} \cdot \frac{\sum_{n=0}^{\infty}(n+1) \frac{a_{n+1}}{a_{0}} \tau^{n}}{\sum_{n=0}^{\infty} \frac{a_{n}}{a_{0}} \tau^{n}} .
$$

It should be noticed that if we define

$$
\hat{a}_{k}=\frac{a_{k}}{a_{0}}, \quad k \geq 0
$$

all $\left\{\hat{a}_{k}, k \geq 0\right\}$ can be immediately derived due to the expression

$$
\hat{a}_{k+2}=\frac{\hat{I}_{1}-\hat{I}_{2}-e^{2 b} \sum_{i=1}^{k}\left[(k-i+1)(k-i+2) \hat{a}_{k-i+2} c_{i}\right]}{\left(e^{2 b}+1\right)(k+2)(k+1)}, \quad k \geq 0,
$$

with $\hat{a}_{0}=1, \hat{a}_{1}=0$. Here, $\hat{I}_{1}$ and $\hat{I}_{2}$ can be respectively expressed as

$$
\begin{aligned}
& \hat{I}_{1}=\left\{\left(i \rho \sigma \phi+m_{j}-2 \Delta\right) e^{2 b} \sum_{i=0}^{k}\left[(k-i+1) \hat{a}_{k-i+1} c_{i}\right]+\left(i \rho \sigma \phi+m_{j}+2 \Delta\right)(k+1) \hat{a}_{k+1}\right\}, \\
& \hat{I}_{2}=e^{2 b} q_{0} q_{2} \sum_{i=0}^{k}\left(\hat{a}_{k-i} c_{i}\right)+q_{0} q_{2} \hat{a}_{k} .
\end{aligned}
$$


Therefore, we have finally arrived at the desired result with

$$
D(\tau)=-\frac{1}{q_{2}} \cdot \frac{\sum_{n=0}^{\infty}(n+1) \hat{a}_{n+1} \tau^{n}}{\sum_{n=0}^{\infty} \hat{a}_{n} \tau^{n}}
$$

according to Equation (3.25). As for $C(\tau ; \phi)$, it is calculated from

$$
C(\tau)=\int_{0}^{\tau} k \theta D(t) d t
$$

since $D(\tau ; \phi)$ has been worked out. As stated previously, $f_{j}$ is the conditional characteristic function of $x_{T}$, thus $P_{j}$ can be derived according to the Gil-Pelaez Theorem [13] once $f_{j}$ is obtained as follows

$$
P_{j}=\frac{1}{2}+\frac{1}{\pi} \int_{0}^{+\infty} \operatorname{Re}\left[\frac{e^{-i \phi \ln [K]} f_{j}}{i \phi}\right] d \phi
$$

By now a formula in series expansion for the price of European call options is derived and can be calculated by Equation (3.5).

Once a series solution is obtained, it is natural for us to consider its convergence. Although the convoluted and recursive structure of Equation (3.27) posed an obstacle when we tried to prove its convergence theoretically, we finally figured out an alternative way. In fact, the series solution is introduced when we seek solutions to ODE (3.13) and thus our final formula will converge if this series solution converges. Therefore, we provide the radius of convergence for the series solution in the following proposition.

Proposition 3.1 The series solution $u=\sum_{n=0}^{\infty} a_{n} \tau^{n}$ will always converge if

$$
\tau \leq \frac{1}{\Delta} \sqrt{b^{2}+\frac{\pi^{2}}{4}}
$$

Proof. According to one existing theory that the radius of convergence of the series solution to a second order linear ODE near an ordinary point is at least as large as the distance from the ordinary point to the nearest singularity of the ODE [4], all we need to 
do for our model is to find the nearest singularity to zero of (3.13). Now if we let

$$
\begin{aligned}
& F_{1}(\tau)=\left(i \rho \sigma \phi+m_{j}\right)\left(e^{2(\Delta \tau+b)}+1\right)-2 \Delta\left(e^{2(\Delta \tau+b)}-1\right) \\
& F_{2}(\tau)=e^{2(\Delta \tau+b)}+1
\end{aligned}
$$

it is not difficult to verify that $F_{1}(\tau)$ and $F_{2}(\tau)$ are both analytic in the entire complex domain. Furthermore, as we notice that $q_{1}$ equals $F_{1}(\tau) / F_{2}(\tau)$ and $q_{0} q_{2}$ is a constant, all singularities can be obtained when we set $F_{2}(\tau)=0$ and are specified as follows

$$
\tau_{k}=-\frac{b}{\Delta}+i \frac{(2 k+1) \pi}{2 \Delta}, \quad k=0,1,2 \ldots
$$

Therefore, the nearest singularity to zero is $-\frac{b}{\Delta}+i \frac{\pi}{2 \Delta}$ and it is actually a simple pole. This implies that the radius of convergence is at least $\frac{1}{\Delta} \sqrt{b^{2}+\frac{\pi^{2}}{4}}$, which has completed the proof.

Except the radius of convergence, the speed of convergence is also an important factor for the series solution and should be examined. Moreover, we will further check the validity of our pricing formula by comparing European option prices calculated by pricing formula (3.5) with those obtained directly from the SDE (3.2) controlling the dynamics with Monte Carlo simulation and from the PDE (3.3) governing the option price with finite difference methods to further demonstrate the speed of convergence and accuracy. These two issues will be shown in the next Section.

\section{Numerical examples and discussions}

In this section, two different examples will be given respectively to show the convergence and the validity of our pricing formula. Of course, the parameters used are chosen under the restriction (3.31). 
As shown from the derivation process in Section 3 that the obtained pricing formula (3.5) can be expressed as

$$
U(S, t, v)=\lim _{n \rightarrow \infty} G(S, t, v ; n)
$$

where $n$ represents the number of terms we use in calculating the option price, the convergence of our formula can be shown if there exists $N$ and $K \in(0,1)$ such that when $n>N$, the following is valid

$$
|G(S, t, v ; n+2)-G(S, t, v ; n+1)| \leq K|G(S, t, v ; n+1)-G(S, t, v ; n)|,
$$

according to the compression conditions for sequences. As a result, when $G(S, t, v ; n+$ 1) $-G(S, t, v ; n) \neq 0$, the following is the only one that need to be considered to check the convergence of the solution

$$
\lim _{n \rightarrow \infty}\left|\frac{G(S, t, v ; n+2)-G(S, t, v ; n+1)}{G(S, t, v ; n+1)-G(S, t, v ; n)}\right|=0 .
$$

In contrast, when $G(S, t, v ; n+1)-G(S, t, v ; n)=0$ holds for all $n>N$, the solution should be regarded as converged. In the following, the values of $G(S, t, v ; n)$ are all calculated in the order of $10^{-16}$.
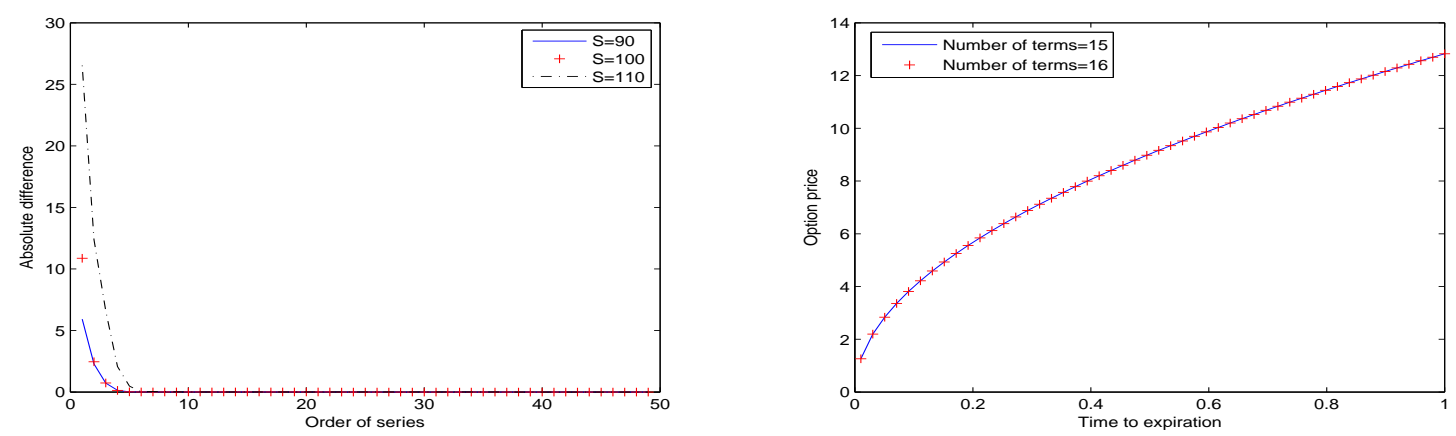

(a) Value of $G(S, t, v ; n+1)-G(S, t, v ; n), 3 \leq n \leq$ (b) Option price when number of terms chosen to 49. be 15 and 16 .

Figure 1: Convergence of pur price in the first example. Parameters are $\rho=-0.5, k=$ $2, \theta=0.1, \beta=0.1, u=4, \tau=0.5, v=0.1, K=100$. 
As is shown in Fig 1(a), the value of $G(S, t, v ; n+1)-G(S, t, v ; n)$ decreases rapidly when $n$ increases and it becomes zero when $n$ reaches 8 , independent of whether the option is "in the money", "at the money" or "out of money". Moreover, it should be noted that the speed of convergence for "in the money" option is slower than that for "out of money" option. To exhibit the convergence of the series solution more clearly, Fig 1(b) displays two option prices corresponding to the cases with 15 terms and 16 terms of the series solution picked respectively when $S$ is set to be 100 . What we can observe first is that the calculated option price is a monotonic increasing function of time to expiration. Furthermore, two different curves are pictured with a difference of $10^{-5}$ order, which can be tolerated when operating in financial markets and thus the 15-term price can be regarded as the converged option price.

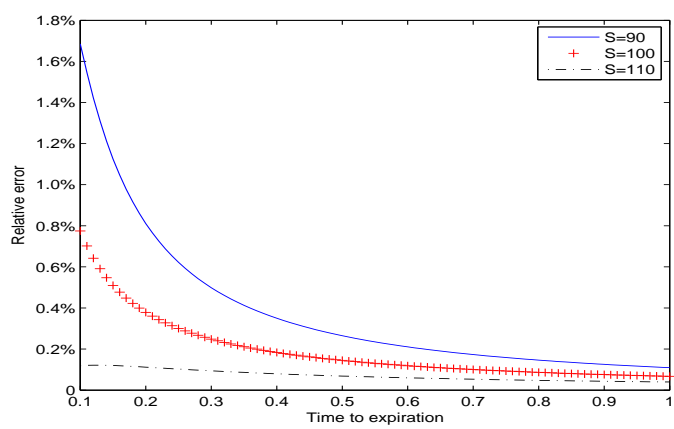

(a) Our price vs finite difference method price.

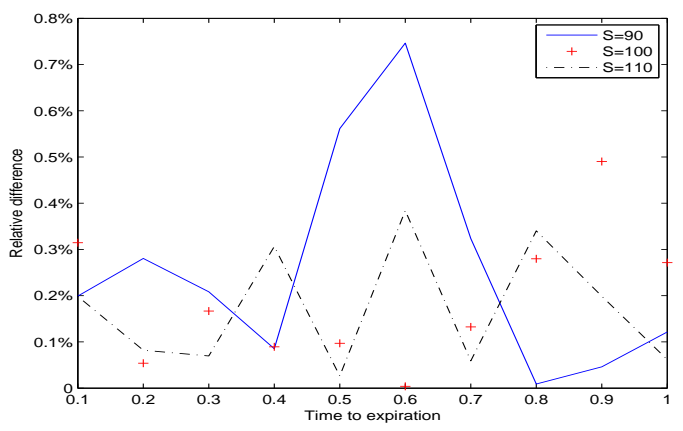

(b) Our price vs Monte Carlo price.

Figure 2: Comparison of our price with those obtained by other numerical methods in the first example. Parameters are $\rho=-0.5, k=2, \theta=0.1, \beta=0.1, u=4, v=0.1, K=100$.

What we can see in Fig 2(a) is the relative difference between our price calculated by 20 terms in the series solution and FDM (finite difference method) price, which is calculated by the explicit scheme. We try to make the results of comparison more convincing by setting $S=90,100,110$ respectively. The maximum difference shown in this sub-figure is less than $1.8 \%$, which could certainly be accepted in real markets. Fig 2(b) further show that our price is quite accurate since there are only no more than $0.8 \%$ difference when our price is compared with Monte Carlo price, which is calculated with 500,000 simulations. When these two sub-figures are viewed together, it should be noticed that prices for "out of 
money" option exhibit higher relative difference than those for options in other positions as expected since prices are smaller for "out of money" call options. On the other hand, as for the speed of calculation, it only takes 2.77 seconds to work out a converged 15 -term price with the closed-form solution, while it costs 639.66 seconds for Monte Carlo simulation and 909.21 seconds for finite different method with the same set of parameters. This implies that working with our closed-form formula can be really time-saving compared with those numerical methods.

Since only one example given by the same set of parameters may not be sufficient to lead to reliable conclusions, another example is chosen. The values of parameters are selected to be quite different from those in the first example to show that it converges for a wide range of parameters as long as the parameters are chosen within the radius of convergence (3.31). As a result, we again calculate the value of $|G(S, t, v ; n+1)-G(S, t, v ; n)|$ when $n$ increases from 13 to 49, which is shown in Fig 3(a). It is obvious that our price still converges in either moneyness of options, although the converging rate is slower than that in the first example. It should also be noticed that the converging speed for "in the money" options is slowest. Fig 3(b) is the one showing how close it is for the price of 25-team and 26-term picked in the series of our solution. Apparently, the order of difference is again only $10^{-5}$, which means that prices of more than 25 terms can be seen as converged.
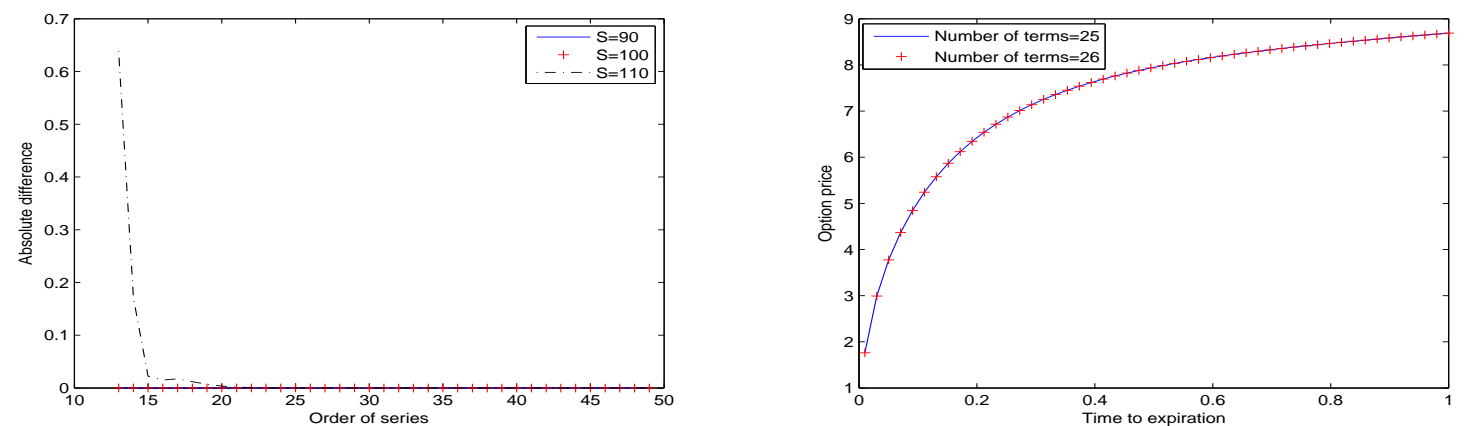

(a) Value of $G(S, t, v ; n+1)-G(S, t, v ; n), 13 \leq n \leq$ (b) Option price when number of terms chosen to 49. be 25 and 26 .

Figure 3: Convergence of pur price in the second example. Parameters are $\rho=0.5, k=$ $5, \theta=0.01, \beta=0.05, u=0.8, v=0.2, \tau=0.5, K=100$. 
Comparison with prices obtained through other numerical methods for this case is also given in Fig 4. This time the relative difference between our price and FDM price is narrowed down to maximum $1.2 \%$ while the distance between our price and Monte Carlo price is still less than $0.8 \%$. Besides, one can still observe that option prices calculated by the closed-form pricing formula have lower relative difference with those obtained through both of the numerical methods when the initial underlying price is no less than the strike price. It should be pointed out that although 25 terms are needed for a converged price, the time it consumes to figure out one price in this case is still very low, at about 4.72 seconds, which is less than $\frac{1}{120}$ of the time that the two numerical methods require. This can significantly raise the efficiency of option pricing in real markets.

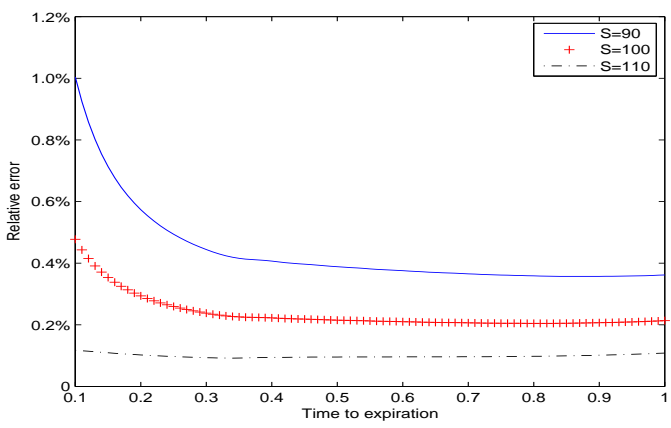

(a) Our price vs finite difference method price.

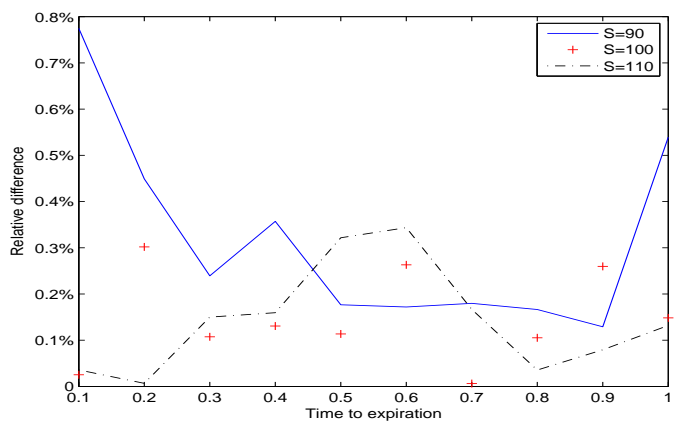

(b) Our price vs Monte Carlo price.

Figure 4: Comparison of our price with those obtained by other numerical methods in the second example. Parameters are $\rho=0.5, k=5, \theta=0.01, \beta=0.05, u=0.8, v=0.2, K=$ 100 .

\section{Conclusion}

In this paper, a closed-form pricing formula for European options is obtained under the minimal entropy martingale measure in the Heston stochastic volatility model with several particular settings. This is a reasonable choice since the specific equivalent martingale measure can be connected with the principle of maximum expected utility. A great advantage of our newly-derived pricing formula is that the convergence of the solution in series form 
can be proved theoretically; such a proof of the convergence has also been complemented by some numerical examples to demonstrate the speed of convergence. Our numerical evidence has further substantiated that the series solution indeed converges very rapidly; when the relative differences with respect to FDM prices and MC prices are maintained to be no more than $1.8 \%$ and $0.8 \%$ respectively, the computational cost associated with adopting the closed-form solution is less than $1 \%$ of those associated with purely numerical methods such as the FDM and MC methods.

\section{Acknowledgements}

The authors gratefully acknowledge constructive comments and suggestions made by the two anonymous referees.

\section{References}

[1] Daily treasury bill rates data. http://www.treasury.gov/resource-center/data-chartcenter/interest-rates/Pages/TextView.aspx?data=billrates.

[2] S. Beckers. Variances of security price returns based on high, low, and closing prices. Journal of Business, pages 97-112, 1983.

[3] F. Bellini and M. Frittelli. On the existence of minimax martingale measures. Mathematical Finance, 12(1):1-21, 2002.

[4] C. M. Bender and S. A. Orszag. Advanced Mathematical Methods for Scientists and Engineers I. Springer Science \& Business Media, 1999.

[5] F. Black and M. Scholes. The pricing of options and corporate liabilities. The journal of political economy, pages 637-654, 1973. 
[6] P. W. Buchen and M. Kelly. The maximum entropy distribution of an asset inferred from option prices. Journal of Financial and Quantitative Analysis, 31(01):143-159, 1996.

[7] S. R. De Groot and P. Mazur. Non-equilibrium thermodynamics. Courier Corporation, 2013.

[8] F. Delbaen, P. Grandits, T. Rheinländer, D. Samperi, M. Schweizer, and C. Stricker. Exponential hedging and entropic penalties. Mathematical finance, 12(2):99-123, 2002.

[9] E. Derman and I. Kani. Riding on a smile. Risk, 7(1):32-39, 1994.

[10] B. Dumas, J. Fleming, and R. E. Whaley. Implied volatility functions: Empirical tests. The Journal of Finance, 53(6):2059-2106, 1998.

[11] B. Dupire. Pricing and hedging with smiles. Mathematics of derivative securities. Dempster and Pliska eds., Cambridge Uni. Press, 1997.

[12] M. Frittelli. The minimal entropy martingale measure and the valuation problem in incomplete markets. Mathematical finance, 10(1):39-52, 2000.

[13] J. Gil-Pelaez. Note on the inversion theorem. Biometrika, 38(3-4):481-482, 1951.

[14] P. S. Hagan, D. Kumar, A. S. Lesniewski, and D. E. Woodward. Managing smile risk. 70+ DVDs FOR SALE \& EXCHANGE, page 249, 2002.

[15] S. L. Heston. A closed-form solution for options with stochastic volatility with applications to bond and currency options. Review of financial studies, 6(2):327-343, 1993.

[16] D. Hobson. Stochastic volatility models, correlation, and the q-optimal measure. Mathematical Finance, 14(4):537-556, 2004. 
[17] J. Hull and A. White. The pricing of options on assets with stochastic volatilities. The journal of finance, 42(2):281-300, 1987.

[18] A. Ilhan, M. Jonsson, and R. Sircar. Optimal investment with derivative securities. Finance and Stochastics, 9(4):585-595, 2005.

[19] N. Krichene. Islamic Capital Markets: Theory and Practice. John Wiley \& Sons, 2012.

[20] J. P. Laurent and H. Pham. Dynamic programming and mean-variance hedging. Finance and stochastics, 3(1):83-110, 1999.

[21] A. Peiro. Skewness in financial returns. Journal of Banking EG Finance, 23(6):847-862, 1999.

[22] G. C. Philippatos and C. J. Wilson. Entropy, market risk, and the selection of efficient portfolios. Applied Economics, 4(3):209-220, 1972.

[23] S. T. Rachev, C. Menn, and F. J. Fabozzi. Fat-tailed and skewed asset return distributions: implications for risk management, portfolio selection, and option pricing, volume 139. John Wiley \& Sons, 2005.

[24] M. Rubinstein. Implied binomial trees. The Journal of Finance, 49(3):771-818, 1994.

[25] M. Schweizer. On the minimal martingale measure and the möllmer-schweizer decomposition. Stochastic analysis and applications, 13(5):573-599, 1995.

[26] M. Schweizer. Approximation pricing and the variance-optimal martingale measure. The Annals of Probability, 24(1):206-236, 1996.

[27] M. Schweizer. A minimality property of the minimal martingale measure. Statistics $\&$ probability letters, 42(1):27-31, 1999. 
[28] L. O. Scott. Option pricing when the variance changes randomly: Theory, estimation, and an application. Journal of Financial and Quantitative analysis, 22(04):419-438, 1987.

[29] S. E. Shreve. Stochastic calculus for finance II: Continuous-time models, volume 11. Springer Science \& Business Media, 2004.

[30] C. Smith. Option strategies: profit-making techniques for stock, stock index, and commodity options, volume 362. John Wiley \& Sons, 2008.

[31] E. M. Stein and J. C. Stein. Stock price distributions with stochastic volatility: an analytic approach. Review of financial Studies, 4(4):727-752, 1991.

[32] S. F. Stoikov. Pricing options from the point of view of a trader. International Journal of Theoretical and Applied Finance, 9(08):1245-1266, 2006.

[33] J. Xia and J. Yan. The utility maximization approach to a martingale measure constructed via esscher transform. preprint, 2000.

[34] S.-P. Zhu and W.-T. Chen. A predictor-corrector scheme based on the adi method for pricing american puts with stochastic volatility. Computers $\&$ Mathematics with Applications, 62(1):1-26, 2011. 